InnOvaciOnes de NegOciOs 16(32): 332-354

(c) 2019 UANL, Impreso en México (ISSN: 2007-1191)

Recepción: 30 Mayo 2019 Aceptación: 18 Junio 2019

\title{
Los adolescentes empacadores del sureste de Tamaulipas y su satisfacción laboral (The adolescent packers of southeast Tamaulipas and their job satisfaction)
}

\author{
María Aurelia Bocanegra Noriega, Mónica Blanco Jiménez, Abel Partida \\ Puente, Pablo Guerra Rodríguez \\ Universidad Autónoma de Tamaulipas, Universidad Autónoma de Nuevo León \\ aureliab@docentes.uat.edu.mx, moniblancoj77@hotmail.com, \\ abel.partidap@uanl.mx, pablo.guerrard@uanl.mx
}

\begin{abstract}
One of the areas of influence of the work focused on the role and conformation of the company, which is evolving along with human societies, one example of this has been manifested in the last half of the last century, when suddenly we went to buy from the small corner store to the big supermarkets, and with it appeared the figure of the merchandise packer (cerillo). This research is descriptive and correlational, causal and transversal exploratory, whose objective is to determine if the elements such as motivation at work, collaboration with their co-workers, economic remuneration, the level of commitment, leadership in supervision affect the job satisfaction of Los teenage packers from the self-service stores in southeast Tamaulipas, using mathematical modeling from Herzberg's theory
\end{abstract}

Key words: Job Satisfaction, adolescent packers.

JEL: J24, M12.

Resumen: Una de las áreas de influencia del trabajo ha incidido en el rol y la conformación de la empresa, la cual a evolucionando a la par que las sociedades humanas, uno ejemplo de ello se ha manifestado en la última mitad del siglo pasado, cuando repentinamente pasamos a comprar de la tiendita de la esquina a los grandes supermercados, y con ello apareció la figura del empacador de mercancías (cerillo). Esta investigación es exploratoria descriptiva y correlacional, causal y transversal, cuyo el

\section{Los adolescentes empacadores del sureste de Tamaulipas}


objetivo es determinar si los elementos como la motivación en el trabajo, la colaboración con sus compañeros de trabajo, la remuneración económica, el nivel de compromiso, el liderazgo en la supervisión inciden en la satisfacción laboral de los adolescentes empacadores de las tiendas de autoservicio del sureste de Tamaulipas, usando la modelación matemática a partir de la teoría de Herzberg.

Palabras Clave: Satisfacción Laboral, adolescentes empacadores.

\section{Introducción}

En la segunda década de este tercer milenio, sigue asombrando la capacidad del hombre para cambiar el entorno que le rodea y ponerlo a su servicio, como resultado de las aplicaciones científicas y tecnológicas generadas por el desarrollo del conocimiento, lo cual ha originado nuevas necesidades acordes a los cambiantes estereotipos sociales. Es aquí donde las empresas juegan un papel protagónico, como satisfactores en gran medida de estas nuevas necesidades. De estas necesidades se ha derivado el incremento del consumo de abarrotes, las empresas de este sector han ido evolucionando enormemente, un ejemplo de ello se ha manifestado en la última mitad del siglo pasado, cuando repentinamente se pasó de comprar de la tiendita de la esquina a los grandes supermercados, simplificando en gran medida las compras y el pago de servicios por parte de las amas de casa y el público en general (ANTAD. 2015).

En el siglo pasado se dieron las condiciones económicas y sociales propicias para que este tipo de negocios se extiendan a lo largo del territorio en México, incidiendo positivamente en la generación de empleos, tanto de personal administrativo, como despachadores, cajeros,

\section{Bocanegra, A., Blanco, M. \& Partida, P.}


de limpieza y sobre todo surge una nueva figura: los adolescentes empacadores, cuyo labor fundamental ha consistido en empacar las mercancía de los clientes en la zona de cajas, a los cuales en este trabajo de investigación se les llamarán adolescentes empacadores (Balderas. 2013).

Dado que este tipo de trabajo de los adolescentes es una actividad muy común, ha sido poco estudiada en otras disciplinas, sin embargo no se han encontrado trabajos que lo relacionen con la satisfacción laboral, por lo que nace esta propuesta de investigación con la inquietud de acercarse a esta figura, el objetivo de esta investigación es medir las variables que pueden tener una incidencia con la la satisfacción laboral de los adolescentes empacadores que trabajan en los supermercados en el sureste de Tamaulipas.

Los datos proporcionados por el Instituto de Estadística Geografía e Informática (INEGI, 2015) reportan sobre la situación del trabajo de los adolescentes empacadores. Estos estudios, son de carácter general y los estudios sobre este tema se ubican en zonas urbanas como son la ciudad de México, Guadalajara Baja California, pero no refieren a la satisfacción laboral, por lo cual surge la siguientes pregunta de investigación de esta investigación: ¿Cuáles son los factores que inciden en la satisfacción laboral de los adolescentes empacadores de las tiendas de autoservicio en el noreste de México? El estudio se localiza en el estado de Tamaulipas, México, en las ciudades del sureste de Tamaulipas. Como hipótesis se tratará de comprobar que los factores que inciden en esta satisfacción son: la motivación en el trabajo, colaboración con sus compañeros de trabajo, remuneración económica,

\section{Los adolescentes empacadores del sureste de Tamaulipas}


nivel de compromiso, y liderazgo en la supervisión, basados en la perspectiva teórica de Herzberg.

En el 2015, el INEGI registro en Tamaulipas una población de 3, 441, 698 habitantes, concentrándose en su mayor parte en 9 municipios que sobrepasan los 100,000 habitantes. En el estado, la distribución demográfica es más densa en tres zonas geoeconómicas: la frontera, el centro y el sureste (INEGI, 2015), en donde las empresas del sector comercial sustentan en gran manera la economía del estado. Respecto al tema del empleo, el INEGI en el último censo del 2015, detectó cerca de 85 mil niños menores de 15 años laborando en diferentes actividades en este estado. Es frecuente la presencia de los menores laborando en las tiendas comerciales, pero sobre todo en las departamentales y las llamadas de autoservicio en donde trabajan empacando las cosas en bolsas y ayudando a las cajeras con los clientes.

A este tipo de jóvenes trabajando se les conoce como los cerillos (empacadores adolecentes) y aunque no son sujetos de un contrato de trabajo, si se les exige una solicitud de empleo y autorización de los padres para que ellos trabajen, así como comprobantes de su situación escolar para acudir a laborar en la empresa, tienen un horario de trabajo que cumplir y cuando faltan tiene que justificarlo, cumpliendo en parte con los apartados en materia laboral aplicables a los menores trabajadores. Su salario son las propinas de los clientes que varían dependiendo de la temporada del año, lo que les permite contribuir a la economía familiar, así como bonos y recompensas que varían según las políticas de la empresa que se trate (Curiel \& Sánchez, 2014).

Bocanegra, A., Blanco, M. \& Partida, P. 
En todo México se observa una fuerte presencia de adolescentes laborando con las características de los cerillos en las tiendas de autoservicio, al igual que en Estado de Tamaulipas, de allí nace la inquietud por conocer la manera como perciben su trabajo a través de la satisfacción laboral.

En México, según INEGI 2015, los jóvenes conforman más del $50 \%$ de la población, ellos poseen perspectivas y opiniones importantes que a menudo no son escuchadas. La participación laboral da poder a los jóvenes y juega un papel vital no solo en su futuro desarrollo, sino también en el cambio social, en el crecimiento económico y en los temas de innovación tecnológica. (UNICEFF, 2011). Ravi Karkara (2011), especialista en Participación de Niños y Adolescentes de la UNICEF, destacó la necesidad de incluir las opiniones de los jóvenes para determinar los métodos de aprendizaje más eficaces: "Ios asuntos de la juventud no llegarán alto en el radar sin la participación de los jóvenes". Para Prateek Awasthi, especialista en programas con niños y adolescentes del mismo organismo expresó: "No es que los jóvenes necesiten aprender a trabajar con adultos, sino que los adultos necesitan aprender a trabajar con los jóvenes". En el caso de los empacadores resulta interesante conocer su opinión de la satisfacción que deriva de su trabajo.

\section{Los adolescentes empacadores del sureste de Tamaulipas}




\section{Marco teórico}

La satisfacción laboral como variable dependiente

La satisfacción laboral es uno de los temas de mayor de interés en el comportamiento organizacional, donde el factor humano se constituye como el elemento más importante del sector productivo, un gran números de autores apoyados en Herzberg, señalan que la satisfacción laboral describe un estado de evaluación que expresa satisfacción y sentimientos positivos sobre el puesto de trabajo, y refiere a dos componentes pertinentes a saber: afectivos que engloban los sentimientos hacia el puesto de trabajo y una parte cognitiva que deriva en una evaluación cognitiva de un puesto de trabajo y sus componentes (Miaoa, Humphreyb, \& Qianc, 2016 ). A partir de lo mencionado, para fines de este trabajo, se puede vincular la satisfacción laboral de los adolescentes empacadores de las tiendas de autoservicio del noreste de México con la teoría de Herzberg, adoptando factores higiénicos e intrínsecos, a través de una relación positiva que resulta de la motivación en el trabajo, la colaboración con sus compañeros de trabajo, la remuneración económica, el nivel de compromiso y el liderazgo en la supervisión.

En cuanto a la medición de la satisfacción laboral, hay varios estudios empíricos que la han medido en varias dimensiones. Una de ellas es el impacto del liderazgo sobre variables como la motivación, satisfacción y clima laboral encontrando que está fuertemente mediada por el grado de supervisión (Cuadra, 2010), también la relacionan con variables como; sueldos y beneficios marginales, condiciones de trabajo

\section{Bocanegra, A., Blanco, M. \& Partida, P.}


y relaciones con sus compañeros de trabajo (Cancio E. J., 2013). En países anglosajones se describe la satisfacción laboral experimentada por trabajadores, concluyendo que el tipo de empleo es la principal determinante que define el grado de la satisfacción experimentada por los trabajadores (Wilkin, 2013).

En E.U se observa una relación positiva entre este constructo y la responsabilidad del trabajo en 203 maestros de áreas rurales de este país (Berry, 2013). En Asia en la ciudad de Teheráse realizó un estudio a 51 empleados de un hospital, relacionando positivamente la satisfacción laboral con los salarios, supervisión, políticas de la organización y condiciones de trabajo (Mohammed, 2015). En Canadá se encuentra una relación positiva en las variables ingreso económico y ambiente laboral con la satisfacción laboral experimentada por pediatras, (Sherif, 2014). En Suiza se realizó un estudio a adolescentes trabajadores y se encontró que la motivación incide en el compromiso laboral pero no en la satisfacción (Hirchi, 2016).

En China se encontró como determinantes de la satisfacción en el trabajo, la satisfacción con la promoción, compañero de trabajo, y la supervisión, presentándose un impacto en ella (Fu, 2014). En México, se estudian a empleados mineros encontrando una relación positiva en la satisfacción laboral y sueldos/salarios, promoción, liderazgo beneficios monetarios y no monetarios, recompensas y reconocimientos, políticas y procedimientos, compañeros de trabajo, el trabajo en sí, comunicación y condiciones del área de trabajo (Peña M. , 2015). En Hamburgo, Alemania en una investigación a jóvenes aprendices de enfermería, se encuentra que es mínima la relación entre la depresión, stress y la

\section{Los adolescentes empacadores del sureste de Tamaulipas}


satisfacción laboral (Kozak T. , 2016). Se observa en estos estudios empíricos realizados diversos contextos geográficos y laborales, donde emergen trabajos que requieren de un nivel de estudio avanzado, hasta aquellos que no requieren ningún grado de especialización, porque son tareas rutinarias y sencillas, así como trabajadores de diferentes edades (Sánchez \& Sánchez, 2018), la presencia de las variables; salario, colaboración, liderazgo, nivel de compromiso, motivación como insumos de entrada para determinar la satisfacción laboral, los cuales pueden ser referenciados en la satisfacción laboral de los adolescentes empacadores del sureste de Tamaulipas.

Motivación en el trabajo

La motivación ha estado presente en estudios que refieren a la satisfacción laboral (Fernet, Gagne, \& Austin, 2010; Kozak, 2016; Hirschi, 2016; Li \& Zhanhong, 2014; Olagoke \& Gamuchirai, 2016). En base a lo referenciado por estos artículos, la motivación se puede entender como una determinante como una disposición psicológica del sujeto hacia su trabajo, que condiciona la satisfacción laboral, estableciendo una relación directamente proporcional, y refiere como un elemento de entrada en la satisfacción laboral de los adolescentes empacadores, de esta forma se puede establecer la siguiente hipótesis: "La motivación se relaciona positivamente con la satisfacción laboral de los adolescentes empacadores de las tiendas de autoservicio del sureste de Tamaulipas."

Bocanegra, A., Blanco, M. \& Partida, P. 
Colaboración con sus compañeros de trabajo

En cuanto a la variable colaboración con sus compañeros de trabajo, la refieren como elemento de entrada en la satisfacción laboral diversos autores, (Pérez \& Azzollini, 2013; Fu \& Deshpande, 2014; Myronaki \& Antonakas, 2009; Oshagbemi, 2013; Paille, Mejía, Marché, Chieh, \& Yang, 2016; Fu W. , 2014). La colaboración es la actitud que tiene un trabajador hacia sus compañeros de trabajo y la satisfacción laboral se puede entender como un conjunto de actitudes generales del empleado hacia su entorno laboral, lo que permite establecer la siguiente hipótesis: "La colaboración con los compañeros de trabajo incide positivamente en la satisfacción laboral de los adolescentes empacadores de las tiendas de autoservicio del sureste de Tamaulipas."

Remuneración económica

La remuneración económica lo refieren la literatura como dato de entrada en la satisfacción laboral, ya que de ella depende el trabajador para cubrir necesidades básicas y de recreación, vinculada esta variable a la teoría de Maslow (Arias, 2013; Cox, 2001; Hosie, 2014; Peña, Díaz, \& Carrillo, 2015; Sherif, 2014; Cancio, 2013; Mohammed, 2015) .Uno de los aspectos más importantes para los empleados es la remuneración que recibe por su trabajo, sin duda ello le permite satisfacer sus necesidades, pero además son un incentivo para ser productivos y leales a la empresa, incentivando la economía de cualquier región del mundo. Así se puede relacionar el ingreso económico con la satisfacción laboral en esta investigación de la siguiente manera: "La remuneración económica se

\section{Los adolescentes empacadores del sureste de Tamaulipas}


relaciona positivamente con la satisfacción laboral de los adolescentes empacadores de las tiendas de autoservicio del sureste de Tamaulipas".

Nivel de compromiso

El concepto de compromiso en el ámbito laboral, se identifica en las organizaciones con un vínculo de lealtad, que lleva al trabajador a permanecer en la organización, conocido también como las actitudes de los empleados en su trabajo, que hace poco probable que el empleado abandone la empresa, siendo la satisfacción laboral un resultado del nivel de compromiso alcanzado por ellos (Ruiz, 2013; Reynaldos \& Cardona, 2014; Alonderine, 2016; Ruiz, 2013; Berry, 2013; Hirschi, 2016). De lo mencionado en esta subsección se entiende la importancia del compromiso que el trabajador manifieste a la empresa, el cual se puede medir de manera positiva y negativa, lo cual va influir en su desempeño laboral, en base a esto se puede considerarlo como un elemento de entrada en la satisfacción laboral, a través de la siguiente hipótesis: "El nivel de compromiso incide positivamente en la satisfacción laboral de los adolescentes empacadores de las tiendas de autoservicio del sureste de Tamaulipas"

\section{Liderazgo}

La palabra liderazgo, refiere a una influencia que se ejerce sobre las personas y que permite incentivarlas, para que trabajen en forma entusiasta por un objetivo común, y el líder es quién ejerce el liderazgo, de allí la importancia de esta figura en las sociedades humanas, la cual

Bocanegra, A., Blanco, M. \& Partida, P. 
ha ido evolucionando a la par de la misma, y en las organizaciones es un elemento fundamental que conlleva a la cultura colaborativa dentro de la empresa como un marco de referencia que ayude al cumplimiento de los objetivos organizacionales, (Barroso \& Salazar, 2010; Chiavenato, 2009; Hannah, Sumanth, \& Lester, 2014; Thompson, 2012; Alonderine, 2016; Contreras \& Cardona, 2015; Horn \& Murillo, 2016; Flecha, 2016). Lo anterior mencionado en los artículos dan cuenta que el liderazgo y la supervisión están relacionados, de manera que; un liderazgo se puede establecer sin ser necesariamente un líder, pero un buen supervisor deben contar con capacidades de liderazgo y cualidades efectivas de supervisión, en el caso de los adolescentes empacadores, se relaciona una buena supervisión con liderazgo, lo cual permite enunciar la siguiente hipótesis: "El que exista un liderazgo en la supervisión incide positivamente en la satisfacción laboral de los adolescentes empacadores del sureste de Tamaulipas "

\section{Metodología}

Con el fin de cumplir con el objetivo general de investigación propuesto, se consideró limitar el trabajo de campo a las tiendas autoservicio con gran área de influencia en la región, ya que se desarrolla la actividad laboral suficiente para garantizar resultados en la satisfacción laboral de los adolescentes empacadores de la zona de Tampico- Madero, ubicados en el sureste de Tamaulipas. Esta investigación es exploratoria descriptiva y correlacional, causal y transversal, cuyo el objetivo es determinar si los elementos como la motivación en el trabajo, la colaboración con sus compañeros de trabajo, la remuneración

\section{Los adolescentes empacadores del sureste de Tamaulipas}


económica, el liderazgo en la supervisión inciden en la satisfacción laboral de los adolescentes empacadores de estas tiendas, se realizó en primer lugar el análisis del Alpha Cronbach a las observaciones realizadas, para verificar la confiabilidad del instrumento, enseguida se hizo un análisis estadístico del perfil del egresado con las primeras 11 preguntas del instrumento, para proceder al análisis de correlación entre las dimensiones resultantes y para finalizar se formuló un análisis de regresión de las variables explicativas propuestas.

\section{Participantes}

Con base a lo planteado para la investigación, la población la constituyó los adolescentes empacadores de las tiendas de autoservicio; Arteli, Chedraui, Soriana, HBE. Walt Mart y Bodega Aurrera de la zona TampicoMadero. La muestra fue seleccionada aleatoriamente, con un nivel de confianza del $95 \%$ y un margen de error de 0.05 , el tamaño de la muestra fue de 187 adolescentes logrando 191 observaciones. La muestra final se compuso de 73 varones y 118 mujeres, de los cuales la mayoría estudian secundaria, laboran en promedio 4 horas diarias durante 5 días a la semana y presentan una antigüedad de 4.6 meses en promedio. La mayoría vive con sus padres y tienen 2 hermanos.

Medidas de las variables

En la literatura, se encontraron, que los instrumentos más usados en estudios que refieren a la satisfacción laboral en diferentes contextos laborales y geográficos son: El Job Descriptive Index (JDI), El Minnesota Satisfaction Questionnaire (MSQ,), el cuestionario de Meyer y Allen

Bocanegra, A., Blanco, M. \& Partida, P. 
(1991), los cuestionarios desarrollados por Meliá y Peiró (1989)y el de Overall Job Satisfaction de Warr, Cook y Wall (1979), los cuales ya fueron validados. A partir de estos se elaboró el instrumento con 11 preguntas que refieren al perfil del adolescente empacador y 42 preguntas para evaluar las variables explicativs en una escala Likert por medio de 5 opciones que va desde muy insatisfecho a muy satisfecho.

\section{Resultados}

Alpha de Cronbach

El instrumento de medición se sometió al Alpha de Cronbach, las dimensiones propuestas fueron aceptadas como se aprecia en la tabla1. Cumpliendo con criterios de Hair et al. (1999) teniendo como límite inferior .60 y limite superior .90, En el caso de las variables explicativas, se observa que cumplen con este criterio lo que nos habla de la confiabilidad del instrumento.

\begin{tabular}{cc}
\multicolumn{2}{c}{ Tabla 1. Alpha de Cronbach } \\
\hline Variable & Alpha de Cronbach \\
\hline Motivación en el trabajo & 0.766 \\
Colaboración con sus compañeros & 0.840 \\
Remuneración económica & 0.759 \\
Nivel de compromiso & 0.803 \\
Liderazgo en la supervisión & 0.856 \\
Satisfacción laboral & 0.803 \\
\hline
\end{tabular}

Fuente: Elaboración propia a partir del procedimiento Alpha de Cronbach

\section{Los adolescentes empacadores del sureste de Tamaulipas}




\section{Análisis de correlación}

En la tabla. 2 se aprecia el coeficiente de correlación de Pearson en cada una de las variables propuestas, resultando ser todos positivo y mayores de 0.600 denotando un buen grado de correlación, la literatura nos dice que es una correlación positiva, si el coeficiente es 1 es una correlación positiva perfecta (Hair et al., 1999).

Tabla 2. Coeficiente de Correlación de variables propuestas

\begin{tabular}{ccc}
\hline \multicolumn{2}{c}{ Dimensiones } & $\begin{array}{c}\text { Satisfacción en el } \\
\text { trabajo }\end{array}$ \\
\hline $\begin{array}{c}\text { Motivación en el trabajo } \\
\text { Colaboración con sus } \\
\text { compañeros de trabajo } \\
\text { Remuneración } \\
\begin{array}{c}\text { económica } \\
\text { Nivel de compromiso } \\
\text { Liderazgo en la } \\
\text { supervisión }\end{array}\end{array}$ & $\begin{array}{c}\text { Correlación de Pearson } \\
\text { Correlación de Pearson }\end{array}$ & .696 \\
& Correlación de Pearson & .602 \\
\hline
\end{tabular}

Fuente: Elaboración propia a partir del Procedimiento correlaciones bivariadas de SSPS

En la tabla 3, se aprecian los resultados al someter las observaciones al análisis estadístico de regresión, donde el coeficiente de determinación ajustado del modelo $\left(R^{2}\right)$ es de 0.723 , lo cual indica que el modelo explica en un $72.3 \%$ la generación de satisfacción laboral del adolescente empacador. 
Tabla 3. Resumen del modelo

\begin{tabular}{ccccccccc}
\hline $\mathbf{R}$ & $\mathbf{R}^{2}$ & $\begin{array}{c}\mathbf{R}^{2} \\
\text { Ajustad } \\
\mathbf{0}\end{array}$ & $\begin{array}{c}\text { Error } \\
\text { Estanda } \\
\mathbf{r}\end{array}$ & $\begin{array}{c}\text { Cambi } \\
\mathbf{0} \\
\mathbf{R}^{2}\end{array}$ & $\begin{array}{c}\text { Cambi } \\
\mathbf{~ O ~ F ~}\end{array}$ & $\begin{array}{c}\text { Sig. } \\
\text { Cambi } \\
\mathbf{~} \mathbf{F}\end{array}$ & $\begin{array}{c}\text { Drubin } \\
\text { Watso } \\
\mathbf{n}\end{array}$ \\
\hline 1 & .85 & .73 & .723 & 2.53057 & .730 & 99.954 & .000 & 1.918 \\
& 4 & 0 & & & & & & \\
\hline
\end{tabular}

Fuente: Elaboración propia a partir del Procedimiento regresión lineal de SSPS

Comprobación de hipótesis

Tomando como referencia la tabla 4 , nos muestra la constante de regresión y las variables explicativas con el nivel de significancia alcanzado, así como los coeficientes estandarizados, los cuales reflejan la manera como incide el aumento en cada una de las variables explicativas en la satisfacción laboral de los adolescentes empacadores.

Tabla 4. Coeficientes

\begin{tabular}{|c|c|c|c|c|c|}
\hline \multirow{2}{*}{ Modelo } & \multicolumn{2}{|c|}{$\begin{array}{l}\text { Coeficiente no } \\
\text { estandarizados }\end{array}$} & \multirow{2}{*}{$\begin{array}{c}\text { Coeficientes } \\
\text { estandarizados } \\
\text { B }\end{array}$} & \multirow[t]{2}{*}{$t$} & \multirow[t]{2}{*}{ Sig. } \\
\hline & B & $\begin{array}{c}\text { Erro } \\
\text { S. }\end{array}$ & & & \\
\hline Constante & 1.051 & 1.277 & .130 & .823 & .411 \\
\hline $\begin{array}{l}\text { Motivación en } \\
\text { el trabajo }\end{array}$ & .187 & .085 & .213 & 2.188 & .030 \\
\hline $\begin{array}{l}\text { Colaboración } \\
\text { con sus } \\
\text { compañeros } \\
\text { de trabajo }\end{array}$ & .237 & .058 & .151 & 4.065 & .000 \\
\hline $\begin{array}{l}\text { Remuneración } \\
\text { económica }\end{array}$ & .309 & .099 & .085 & 3.116 & .002 \\
\hline
\end{tabular}




\begin{tabular}{cccccc}
$\begin{array}{c}\text { Nivel de } \\
\text { compromiso } \\
\text { Liderazgo en }\end{array}$ & .093 & .070 & .432 & 1.336 & .183 \\
la supervisión & .440 & .064 & .130 & 6.905 & .000 \\
\hline
\end{tabular}

La tabla 5 denota las hipótesis aceptadas en base a la literatura, la cual dice que los niveles de significancia deben ser iguales o menores a 0.05 para ser estadísticamente significativos (Hair et al., 1999).

Tabla 5. Hipótesis aceptadas

\begin{tabular}{ccc}
\hline Variable explicativa & Significancia & Resultados \\
\hline$X_{1}=$ Motivación en el & 0.030 & \\
trabajo & & \\
$X_{2}=$ Colaboración con sus & 0.000 \\
compañeros de trabajo & \\
$X_{3}=$ Remuneración & 0.020 \\
económica & \\
$X_{4}=$ Nivel de compromiso & 0.183 \\
$X_{5}=$ Liderazgo en la & 0.000 \\
supervisión & & \\
\hline
\end{tabular}

Fuente: Elaboración propia a partir del Procedimiento regresión lineal de SSPS

En base a lo anterior se puede determinar el modelo matemático de la satisfacción de los adolescentes empacadores en la siguiente ecuación:

Bocanegra, A., Blanco, M. \& Partida, P. 


$$
y=1.051+1.87 X_{1}+0.237 X_{2}+0.309 X_{3}+0.440 X_{4}
$$

Con estos resultados se cumple con el objetivo general de este trabajo de investigación.

\section{Conclusiones}

Los resultados permiten comprobar que en este sector laboral específico donde trabajan los adolescentes empacadores en Tampico-Madero, Tamaulipas, se identifica un considerable nivel de satisfacción laboral en las variables explicativas motivación en el trabajo, colaboración con sus compañeros de trabajo, remuneración económica y liderazgo en la supervisión, debido a los niveles de significancia alcanzados en el análisis de regresión, estos resultados concuerdan con los estudios realizados sobre satisfacción laboral en diversos entornos laborales, Fernet, Gagne, \& Austin (2010), Kozak T. ( 2016), Hirschi A. (2016), Olagoke \& Gamuchirai (2016).

En contraste con lo anterior, cabe mencionar que la variable explicativa nivel de compromiso como elemento de entrada en la satisfacción laboral en los empacadores, al no alcanzar el nivel de significancia requerido quedó fuera del modelo matemático, no concordando este resultado con los estudios empíricos realizados en otros ambientes laborales y geográficos Alonderine (2016), Berry (2013), Hirsch ( 2016), y esto es un indicador importante, ya que unos trabajadores identificados con su empresa siempre harán su mejor esfuerzo en sus labores que coadyuven a los objetivos de la organización.

\section{Los adolescentes empacadores del sureste de Tamaulipas}


Esta diferencia nos lleva a la reflexionar: Sí el contexto es diferente, los determinantes que afectan a la satisfacción laboral son también distintos. Lo anterior mencionado da la pauta para investigaciones futuras, en otras empresas del mismo giro, para observar si se repite este mismo comportamiento en los adolescentes empacadores respecto de su satisfacción laboral.

La satisfacción laboral en cualquier ámbito geográfico y laboral es determinante para el desarrollo de las organizaciones y por ende para la sociedad (Zierold, 2016), en el caso específico de este trabajo de investigación reviste de mayor importancia ya que se trata de las primeras experiencias laborales de los adolescentes empacadores no abordadas en la literatura que refieren a este tema, estos adolescentes serán en el corto plazo el capital más importante en el sector productivo, y un mal uso de este capital intelectual, incide de manera negativa en el crecimiento económico en cualquier región del mundo y por el contrario una fortalecida relación laboral incide positivamente en estos aspectos. (Weller, 2018). Este trabajo contribuye a llenar un hueco en al literatura porque permitió conocer la opinión de adolescentes que cotidianamente laboran en las tiendas de autoservicio, y se logró plasmar esta información en un modelo matemático nos congratulamos con los organismos internacionales que dan la pauta para que se lleven a cabo este tipo de trabajos de investigación.

Bocanegra, A., Blanco, M. \& Partida, P. 


\section{Referencias}

Alonderine, R. (2016). Leadership style and job satisfaction in higher education institutions. International Journal of Education Management, 30(1), 140-164. Disponible en: https://doi.org/10.1108/IJEM-08-2014-0106

Asociación Nacional de Tiendas de Autoservicio (2015). Comunicado de prensa. Disponible en: https://antad.net/

Arias, W., Justo. O. (2013). Satisfacción laboral en trabadores de dos tiendas por departamento: un estudio comparativo. Ciencia y trabajo, 15(47), 41-46. Disponible en: https://scielo.conicyt.cl/pdf/cyt/v15n47/art03.pdf

Balderas, E. (2013). El trabajo de los menores en tiendas de autoservicio. Universidad Autónoma Metropolitana.

Barroso, F. (2010). Liderazgo y clima organizacional en maquiladoras textiles de exportación. The Anahuac Journal Business and Economics, 10(2), 67-96

Berry, A (2013). The relationship of perceived support to satisfaction and commitment for special education teachers in rural areas. Rural Special education Quarterly, 31(1), 3-14. Disponible en: https://files.eric.ed.gov/fulltext/EJ1147842.pdf

Cancio, E. J. (2013). Defining Administrative Support and Its Relationship to the Attrition of Teachers of Students with Emotional and Behavioral Disorders.

Education and Treatment of Children, 36(4), 71-94. doi. 10.1353/etc.2013.0035

Chiavenato, I. (2009). Gestión del talento humano. México: McGraww-Hill.

Contreras, V., Reynaldos, K., \& Cardona L. (2005). Clima, ambiente y satisfacción laboral: un desafío para la enfermería. Revista cubana de enfermería, 31(1), 58-69. Disponible en: https://researchers.unab.cl/en/publications/climaambiente-y-satisfacci\%C3\%B3n-laboral-un-desaf\%C3\%ADo-para-laenfermer

Cox, K. B. (2001). The effects of unit morale and interpersonal relations on conflict in the nursing unit. Journal of Advanced Nursing, 35(1), 17-25. Disponible en: https://www.ncbi.nlm.nih.gov/pubmed/11442678

\section{Los adolescentes empacadores del sureste de Tamaulipas}


Cuadra, A. (2010). Grado de supervisión como variable moderadora entre liderazgo. Ingeniere revista Chilena de ingeniería, 18(1), 15-25. Disponible en: https://scielo.conicyt.cl/scielo.php?script=sci_arttext\&pid=S071833052010000100003

Curiel, V., \& Sánchez, J. (2014). Condiciones laborales de los menores empacadores en el Distrito Federal. Revista Jurídica de la Universidad Autónoma Metropolitana, 179-204

Fernet, C., Gagne, M., \& Austin, S. (2010). When does quality of relationships with coworkers predict burnout over time? The moderating role of work motivation. Journal of Managmente organizational Behavior, 1163-1180.

Flecha, G. E. (2016). The perceptions of practical work experience on student motivation to complete a Pharmacy Technician program. Dissertations \& Theses Gradworks, 75(8), 151. doi: 10.5688/ajpe758151

$\mathrm{Fu}, \mathrm{W}$. (2014). The Impact of Emotional Intelligence, Organizational Commitment, and Job Satisfaction on Ethical Behavior of Chinese Employees. Journal of Business Ethics, (1), 137- 144. Disponible en: https://doi.org/10.1007/s10551013-1763-6

Guerra, G. (2012). Políticas de protección laboral en etapas de crisis en México: el Seguro de desempleo del Distrito Federal. Estudios Demográfico y Urbanos, 3(81), 662-708. Disponible en: http://www.scielo.org.mx/pdf/educm/v27n3/2448-6515-educm-27-03-661.pdf

Hannah, S., Sumanth, J., \& Lester, P. (2014). Debunking the false dichotomy of leadership idealism and pragmatism: Critical evaluation and support of newer genre leadership theories . Journal of Organizational Behavior, 35(5), 598-621. Disponible en: https://doi.org/10.1002/job.1931

Hair, J. F, Anderson, R. E; Tatham, R. L; y Black, W. C. (1999). Análisis Multivariante, 5a. Edición, Madrid: Prentice-Hall

Hernández, R., Collado, C., \& Baptista, P. (2016). Metodología de la Investigación. México: Ma Graw Hill

Herzberg, F. (1966). Work and the Nature of Man,. Oxford, Englasnd: APA 
Hirschi, A. (2016). Latent profiles of work motivation in adolescents in relation to work expectations, goal engagement, and changes in work experiences. Journal of vocational behavior, 93, 67-80. Disponible en: https://doi.org/10.1016/j.jvb.2016.01.003

Horn, A., \& Murillo, F. (2016). Incidencia de la dirección escolar sobre el compromiso de los docentes: Un estudio multinivel. Psicoperspectivas: individuo y sociedad. 15(2), 64-77. Disponible en: https://www.psicoperspectivas.cl/index.php/psicoperspectivas/article/view/74 $6 / 500$

Wirth, T., Kozak, A., Schedlbauer, G., \& Nienhaus, A. (2016). Health behaviour, health status, and occupational prospects of apprentice nurses and kindergarten teachers in Germany: a cross-sectional study. Journal of Occupational Medicine and Toxicology, 11(26). doi: 10.1186/s12995-016-0116-7

Li, M., \& Wang, Z. (2014). Emotional labour strategies as mediators of the relationship between public service motivation and job satisfaction in Chinese teachers. International Journal of Psychology, 51(3), 177-184. doi: 10.1002/ijop.12114

Miao, C., Humphrey, R., \& Qian, S. (2016). Leader emotional intelligence and subordinate job satisfaction: a meta-analysis of main, mediator, and moderator effects. Personality and Individual Differences, 102, 13-24. https://doi.org/10.1016/j.paid.2016.06.056

Mohammed, A. (2015). Strategic collaborative quality management. International Journal of Health Policy and Management, 2(4), 167-174. doi: 10.15171//JHPM.2014.38

Myronaki, A., \& Nikolaos, A. (2009). Previous employment and job satisfaction conditions: The case of regional administration. AIP conference proceedings, 1148(1). Disponible en: https://doi.org/10.1063/1.3225471

Olagoke, A., \& Gamuchirai, C. (2016). Factors influencing motivation and job satisfaction among supervisors of community health workers in marginalized communities in South Africa. Human Resources for Helth, 14(54). doi: 10.1186/s12960-016$0151-6$

Oshagbemi, T. (2013). Job Higher Education. Ireland: Trafford Publishing.

\section{Los adolescentes empacadores del sureste de Tamaulipas}


Paille, P., Mejía, J., Marché, A., Chieh, C., \& Yang, C. (2016). Corporate Greening, Exchange Process Among Co-workers, and Ethics of Care: An Empirical Study on the Determinants of Pro-environmental Behaviors at Coworkers-Level. Journal of Business Ethics, 136, 655-673. doi: 10.1007/s10551-015-2537-0

Peña, M., Díaz, M., \& Carrillo, A. (2015). Relación del clima organizacional y la satisfacción laboral en una pequeña empresa familiar. Revista Internacional Administración \& Finanzas, 8(1), 37-50. Disponible en: https://ssrn.com/abstract=2499791

Perez, P., \& Azzollini, S. (2013). Liderazgo, equipos y grupos de trabajo- su relación con la satisfacción laboral. revista de Psicoligía, 31(1) 152-169. Disponible en: https://www.redalyc.org/pdf/3378/337829524006.pdf

Reynaldos, K., \& Cardona, L. (2014). Job climate, work environment and job satisfaction: challenge for nursing. Revista Cubana de Enfermería, 31(1), 58-69. Disponible en:

http://www.scopus.com/inward/record.url?scp=84994523003\&partnerlD=8YF LogxK

Ruiz. S., Reboredo, F., \& Jiménez, G. (2007). Percepción del clima y satisfacción laboral en la empresa de papantla veracruz. Medicina, salud y sociedad. 1(3), 1-18. Disponible en: https://pdfs.semanticscholar.org/1b4e/804d34f52cc0363d4f8a4cec91553f74f 923.pdf

Sánchez-Sellero, M., \& Sánchez-Sellero, P. (2018). Determinantes de la satisfacción laboral en la industria de la madera y el papel: estudio en España y hallazgos en otros países. Maderas, ciencia y tecnología, 20(4), 641-660. https://dx.doi.org/10.4067/S0718-221X2018005041101

Sherif, E. (2014). Satisfaction levels of paediatric surgeons in Canada. Paediatrics \& Child Health, 19(9). 481-484. doi: 10.1093/pch/19.9.481

Thompson, J. (2012). Transformational leadership can improve workforce competencies. Nursing Management, 18(190). 21-4. doi: 10.7748/nm2012.03.18.10.21.c8958

\author{
Bocanegra, A., Blanco, M. \& Partida, P.
}


Fondo de las Naciones Unidas para la Infancia (2011). Participación de los jóvenes, como parte del año internacionl de la juventud. Disponivle en: https://www.un.org/es/events/youth2010/hlm.shtml

Webb, E., Panico, L., Bécares, L., McMunn, A., Kelly, Y., \& Sacker, A. The interrelationship of adolescent unhappiness and parental mental distress. Journal of Adolescence Health, 60(2). 196-203. doi: 10.1016/j.jadohealth.2016.10.001

Weller, J (2018). Aspects of recent developments in the Latin American and Caribbean labour markets. Revista de la Comisión Económica para América Latina y el Caribe. Disponible en:

http://www.cepal.org/publicaciones/xlm//LCG2339eWeller.pdf

Wiess, D., Dawis, R., Enggland, G., \& Lofquist, L. (1967). Minessota Sastifaction Questionaire-Psychometric properties and validation in a population of portuguese hospotal workers. FEP Working papers. Disponible en: http://wps.fep.up.pt/wps/wp471.pdf

Wilkin, C. (2013). I can't get no job satisfaction: Meta-analysis comparing permanent and contingent workers. Journal of organizational behavior, 34(1). 195-224. Disponible en: https://doi.org/10.1002/job.1790

Zierold, K. (2016]). Safety training for working youth: Methods used versus methods wanted. Journal of preventio assessment \& rehabilitation, 54(1). 149-57. doi: 10.3233/WOR-162291.

\section{Los adolescentes empacadores del sureste de Tamaulipas}

